\title{
Use of the Sideguard (Cappella) stent in bifurcation lesions: a real-world experience
}

\author{
Abstract
}

AIMS:

The Sideguard® stent (Cappella Medical Devices Ltd, Galway, Ireland), is a novel nitinol self-expanding dedicated bifurcation stent that flares proximally at the ostium of the side branch (SB) into a trumpet shape thereby achieving full ostial coverage. The aim of this study is to report the utility and limitations of this stent in patients undergoing treatment to bifurcation coronary lesions in a real-world setting.

\section{METHODS AND RESULTS:}

We prospectively identified 20 successive patients admitted over a 6-month period in whom there was significant SB disease and who were suitable for a bifurcation procedure. The Sideguard ${ }^{\circledR}$ stent was successfully used in all 20 cases including several that would have been technically difficult using conventional bifurcation techniques. We highlight use of this system using five illustrative cases that illustrate its utility and limitations in the treatment of bifurcation lesions.

\section{CONCLUSIONS:}

The Sideguard® stent can be used to treat complex bifurcation lesions in a straight forward manner and is not subject to the limitations associated with conventional bifurcation PCI techniques including jailing of the SB ostium and inability to fully cover/scaffold the ostium of the SB. 